\title{
Startup of the high-intensity ultracold neutron source at the Paul Scherrer Institute
}

\author{
Bernhard Lauss \\ on behalf of the PSI UCN Project Team
}

Published online: 2 March 2012

(C) Springer Science+Business Media B.V. 2012

\begin{abstract}
Ultracold neutrons (UCN) can be stored in suitable bottles and observed for several hundreds of seconds. Therefore UCN can be used to study in detail the fundamental properties of the neutron. A new user facility providing ultracold neutrons for fundamental physics research has been constructed at the Paul Scherrer Institute, the PSI UCN source. Assembly of the facility finished in December 2010 with the first production of ultracold neutrons. Operation approval was received in June 2011. We give an overview of the source and the status at startup.
\end{abstract}

Keywords Ultracold neutrons $\cdot$ UCN $\cdot$ Neutron source $\cdot$ Ultracold neutron production and transport

\section{Introduction}

The fundamental properties of the neutron can serve as a window into the first minutes of the universe. The neutron lifetime is an important parameter in understanding the abundance of light elements created in the big-bang nucleo-synthesis [1]. Finding a neutron electric dipole moment (nEDM) [2] would hint at a new CP violating process necessary to understand the asymmetry of matter over antimatter in our universe. nEDM searches (e.g. [3, 4]) probing physics beyond the Standard Model are presently statistics limited and are the main driving force behind the proposals of several new high-intensity ultracold neutron sources around the globe [5]. Moreover, stored neutrons allow to search for extra forces not included in the Standard Model of particle physics or search for modifications of Newtonian gravity in the sub-millimeter range predicted by string theories [6].

The members of the PSI UCN Project Team are listed at http://ucn.web.psi.ch.

B. Lauss $(\varangle)$

Paul Scherrer Institute, 5232 Villigen-PSI, Switzerland

e-mail: bernhard.lauss@psi.ch 


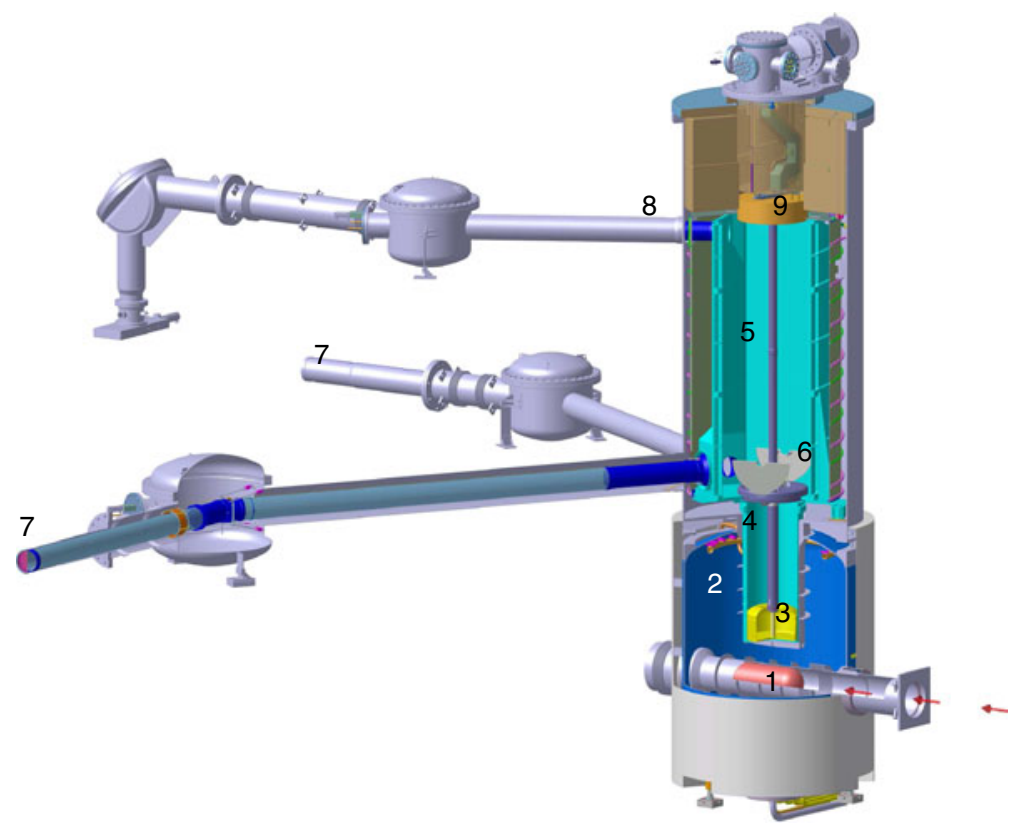

Fig. 1 Cut view of the neutron production, storage and guiding system of the PSI UCN source inside a $7 \mathrm{~m}$ high vacuum tank. The indicated components are described in the text

\section{The ultracold neutron source}

Neutrons with kinetic energies below $\sim 350 \mathrm{neV}$, corresponding to a few milli-Kelvin, are termed ultracold neutrons (UCN). The material optical potential of certain materials (e.g. Ni, Be, steel, diamond-like carbon/DLC) is high enough-due to their high density and large neutron bound-coherent scattering length - that UCN undergo total reflection under all angles of incidence [2]. Hence, UCN can be stored in material bottles for several hundreds of seconds. They can also be contained via gravity, with an energy change of $100 \mathrm{neV}$ per meter and they can be manipulated via their magnetic moment, where a 1 Tesla field change corresponds to a change of $60 \mathrm{neV}$ in potential energy.

Over the last years an ultracold neutron source based on accelerator driven spallation neutron production has been constructed at the Paul Scherrer Institute (PSI), Switzerland [7, 8].

The method, experimentally pioneered at PNPI [9] and Los Alamos National Lab [10], is based on (i) neutron production via proton induced spallation on lead [11]; (ii) superthermal UCN production in solid ortho-deuterium ( $\left.\mathrm{s} D_{2}\right)$ [2]; and (iii) intermediate UCN storage and distribution of UCN on demand to experiments. The main experimental components covering the neutron production and transport are shown in Fig. 1. PSI's $590 \mathrm{MeV}$ proton beam with up to $2.4 \mathrm{~mA}$ beam current is impinging on a heavy-water-cooled canneloni-type lead spallation target (see (1) in Fig. 1) [11]. UCN operation is limited by radiation safety requirements to an integrated proton flux of $20 \mu \mathrm{A} /$ hour. This means that a $2 \mathrm{~mA}$ proton beam kick 

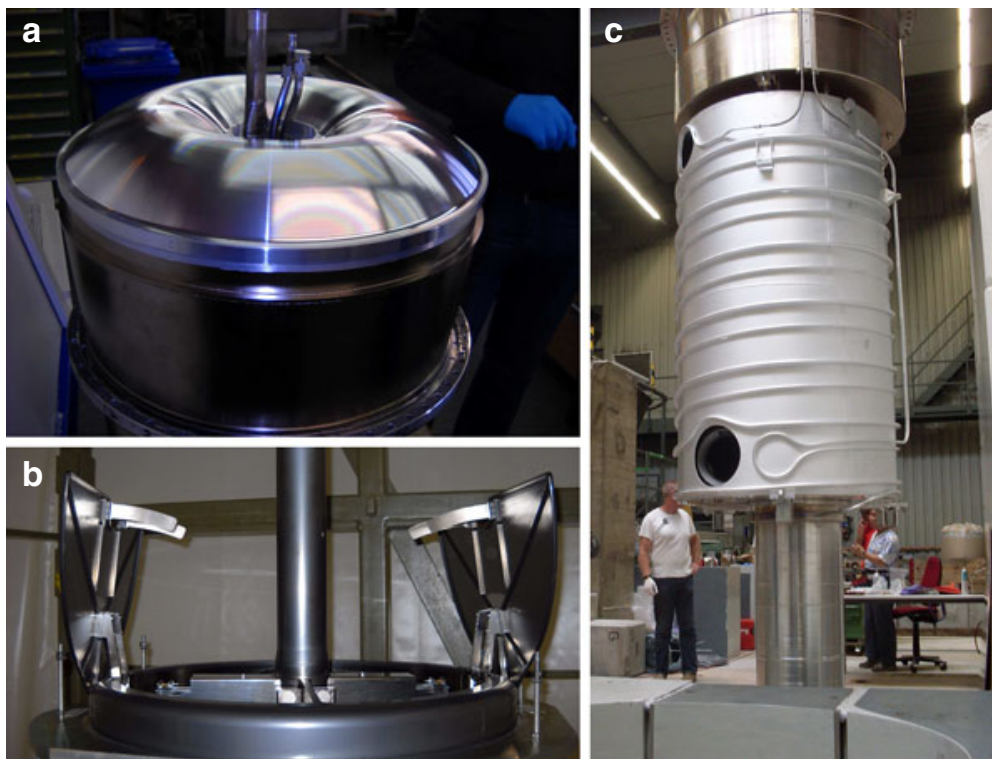

Fig. 2 Parts of the UCN source during assembly. a The container for the solid deuterium crystal. The donut shaped top-lid, necessary for pressure safety, is only $0.5 \mathrm{~mm}$ thick to allow for a high UCN transmission. b The storage volume shutter during operation testing. The 2 aluminum jaws visible on each flap are used for stopping the closing flaps via an eddy-current brake working at $80 \mathrm{~K}$. c The UCN storage volume before insertion in the vacuum tank. Visible is the vertical neutron guide on the bottom, the radiation shield surrounding the UCN storage vessel with the $80 \mathrm{~K}$ cooling tubes and the opening for the neutron guides. The steel shielding is on top

of $8 \mathrm{~s}$ is followed by a minimum waiting period of $800 \mathrm{~s}$. Spallation neutrons are thermalized in the surrounding heavy water tank (2). Close to the spallation target inside a AlMg3 container (No. (3) in Figs. 1 and 2a) a pure $~ 30$ liter ortho-deuterium crystal is kept at a temperature of $5 \mathrm{~K}$. It serves to moderate neutrons first to be cold and finally to be ultracold neutrons via downscattering $[2,5,13]$.

The UCN production rate strongly depends on the deuterium temperature and spin $[5,12,13]$ favoring solid ortho-deuterium as production medium. Some UCN can then be emitted into the vacuum on top of the crystal, where they get a $102 \mathrm{neV}$ boost from the $\mathrm{s} D_{2}$ material potential at the crystal surface. Via a vertical UCN guide (4) (shown in Fig. 2c) they can reach the UCN storage vessel (5) coated with diamondlike carbon which has a high material optical potential of about $235 \mathrm{neV}$ and a very low neutron loss probability.

After the beam kick the main UCN shutter (No. (6) and Fig. 2b) is rapidly closed and the produced UCN are stored inside the vessel. Three UCN guides (7) and (8) lead through the radiation shielding to experimental areas. Shutters can open and close these guides at the storage vessel exits. In order to provide optimal UCN transmission through more than $8 \mathrm{~m}$ of tubes penetrating the radiation shielding, most of the guides are made of $180 \mathrm{~mm}$ inner diameter Duran ${ }^{\circledR}$ glass tubes with approximately $1 \mathrm{~nm}$ surface roughness and sputter-coated with $500 \mathrm{~nm}$ thick NiMo on the inside. A $80 \mathrm{~cm}$ long polished steel guide connects the glass guides at 


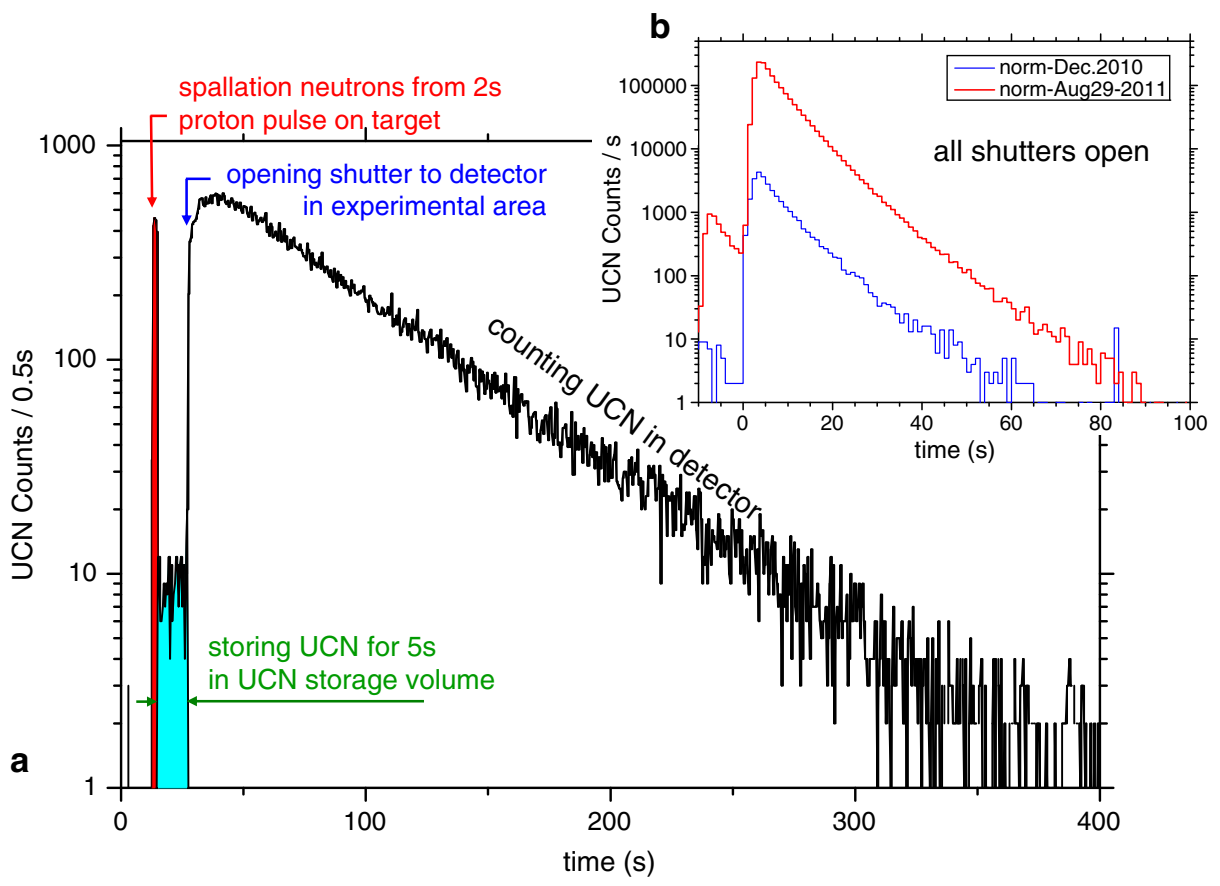

Fig. 3 a First UCN in December 2010: UCN counts observed in a Cascade-U detector located at the end of the UCN guide in area West, plotted versus time (seconds). The structure in the count rate reflects the initial proton beam kick onto the spallation target of $2 \mathrm{~s}$ which produce a neutron background in the detector. After the beam kick the UCN storage volume is closed and the UCN are stored for $5 \mathrm{~s}$. A few percent of the UCN leak during that time through the guide UCN shutter. After $5 \mathrm{~s}$ the guide shutter towards the experimental area is opened and the UCN can arrive at the detector. The observed count rate then reflects the exponential emptying of the UCN storage vessel into the detector. b UCN counts versus time after beam kick observed on guide West-1 for 'normalized pulses', i.e. beam kicks with $2 \mathrm{~s}$ length and all shutters, including the main UCN storage vessel shutter remaining open all the time. Hence the emptying of the storage vessel is determined by the large opening towards the deuterium container. The given time distributions are for the best beam kick observed in Dec. 2010 and the startup kicks in Aug. 2011. A factor 50 improvement is obvious

room temperature to the UCN storage vessel which is operating at $80 \mathrm{~K}$. Radiation protection requires $30^{\circ}$ bends (made from polished stainless steel) to prevent direct sight onto the storage vessel. On top of the UCN storage vessel a large, activated carbon loaded cryo-pump is the coldest spot of the source vacuum in order to efficiently capture residual gas atoms and prevent them from condensing on the s $D_{2}$ container or anywhere else inside the storage vessel.

Several important components are not shown on the figure. Most prominently the cryo-system necessary for the production of the ortho-deuterium crystal. The present $\mathrm{s} D_{2}$ production procedure [14] uses $30 \mathrm{~m}^{3}$ pure $D_{2}$ gas. The gas is frozen into the 'condenser' volume. Then it is liquefied and transported to the nearby 'para-otho-converter' filled with a paramagnetic chromium-oxide (Oxisorb ${ }^{\circledR}$ ) [15] and left boiling at $19 \mathrm{~K}$ for several hours. Raman spectroscopy of the rotational transitions in $D_{2}$ on extracted gas samples shows an ortho-deuterium concentration 
of $97 \pm 2 \%$ as expected for equilibrium conditions at $19 \mathrm{~K}$. Finally, the ortho- $D_{2}$ is slowly frozen inside the crystal container. This process takes several days and, being a very important part of the source setup, still needs to be optimized.

Final assembly of all parts and commissioning of the PSI source finished in 2010. December 16, 2010 saw the first UCN production during testing the radiological and cryogenic safety. Figure 3 a shows one of the first production fills with a UCN countrate structure as expected. Beam kicks of up to $8 \mathrm{~s}$ length and full beam power were tested.

Operation approval of the Swiss Federal Authorities was received on June 27 and the source operation started in August, 2011. The source performance is continuously improving since then with presently already a factor 50 higher yield in comparison to Dec. 2010 as demonstrated in Fig. 3b. This can be mainly attributed to increasing the ortho-deuterium content from 66 to $\sim 97 \%$ and a different freeze-out technique. A factor of about 30 lies ahead to full design intensity.

The experimental search for a neutron EDM is starting in area South with an installed apparatus [3]. The UCN user facility at PSI is open for experiment proposals.

Acknowledgements Cordial thanks to the more than two hundred colleagues contributing to the UCN source project at the Paul Scherrer Institute who are indispensable for the realization of this project. Support of our colleagues at PF2-ILL and the Mainz TRIGA UCN Source during component testing and of Jagellonian University Cracow and LPSC Grenoble is gratefully acknowledged. PNPI contributed in the early planning of the project.

\section{References}

1. Particle Data Group, Nakamura, K., et al.: JPG 37, 075021 (2010)

2. Golub, R., Richardson, D., Lamoreaux, S.K.: Ultra-Cold Neutrons. Adam Hilger, Bristol, Philadelphia and New York (1991)

3. Altarev, I., et al.: Nucl. Instrum. Methods A 611, 133 (2009)

4. Baker, C.A., et al.: Phys. Rev. Lett. 97, 131801 (2006)

5. Kirch, K., et al.: Nucl. Phys. News 20/1, 17 (2010)

6. Abele, H.: Prog. Part. Nucl. Phys. 60, 1 (2008)

7. Blau, B.: In: Proceedings of the ICANS XIX Meeting. ISSN-Nr.1019-6447, Grindelwald, Switzerland, 8-12 Mar 2010

8. Lauss, B.: J. Phys.: Conf. Ser. 312, 052005 (2011)

9. Serebrov, A.P., et al.: JETP Lett. 66(12), 802 (1997)

10. Morris, C.L., et al.: Phys. Rev. Lett. 89, 272501 (2002)

11. Wohlmuther, M., Heidenreich, G.: Nucl. Instrum. Methods A 564, 51 (2006)

12. Liu, C.-Y., et al.: Phys. Rev. B 62(6), R3581 (2000)

13. Atchison, F., et al.: Phys. Rev. C 71, 054601 (2005)

14. Anghel, A., et al.: In: Proc. of the 10th IIR International Conference Cryogenics 2008. Prage, Czech Republic, 21-25 Apr 2008

15. Bodek, K., et al.: Nucl. Instrum. Methods A 533, 491 (2004) 